\title{
Quantitative Expression of Outlet Deviation Angle of Turbomachine Stator Based on Equivalent Moment of Momentum Principle
}

\author{
Wenjia Li ${ }^{1, *}$, Anlin Wang ${ }^{1}$, Wei Cheng ${ }^{1}$, Xiaotian $\mathrm{Li}^{1}$ and Jibin $\mathrm{Han}^{2}$ \\ ${ }^{1}$ School of Mechanical Engineering, Tongji University, 201804, Shanghai, China; \\ ${ }^{2}$ Shantui Construction Machinery Co., Ltd, 272073, Shandong, China \\ Corresponding Email: pigeon_1wj_1989@126.com
}

\begin{abstract}
In order to express outlet deviation angle of turbomachine stator, outlet deviation angle dominantly expressed by fluid velocity distribution was derived based on equivalent moment of momentum under the condition of certain CFD simulation design parameters. On this basis, response surface function of outlet deviation angle was constructed by CFD simulation data of orthogonal experiment. The validity of the response surface function was proved by CFD simulation data of confirmatory models. An effective method is provided for calculating outlet deviation angle of turbomachine stator.
\end{abstract}

Keywords. Equivalent moment of momentum principle, outlet deviation angle, quantitative expression, turbomachine stator, computational fluid dynamic (CFD)

\section{Introduction}

The turbomachine is a series of power machines, which consists of several rotating impellers, and the energy transfers between impellers fixed on the shafts through the fluid. The turbomachine includes pump, fan, steam turbine, hydraulic torque converter and so on. The classical turbomachine design method is based on one-dimension flow theory, as the calculation speed is fast [1]. But the calculation precision is low, because the theory is based on the simplification of three-dimension flow to one-dimension flow, and it's hard to get accurate parameter values, such as the outlet deviation angle of stator. So, it's important to quantitatively research the outlet deviation angle of turbomachine stator.

The effect of the outlet deviation angle was mostly discussed in the papers [2-4]. Some papers discussed the reason that outlet deviation angle was produced and the phenomena of outlet deviation angle. For example, Willi [1] pointed out the reason that outlet deviation angle produced was the bending blade. Hayder [5] discussed the phenomena of wake formation. Padzillah [6] found the flow angle swings over a wide range in pulsating flow field. But the relationship between flow angle and design parameter was not studied in these papers. Nishi and Fukutomi [7] researched effect of outlet blade angle on outlet flow angle. But the effect of inlet blade angle and flow rate on outlet flow angle was not considered.

In this paper, equivalent moment of momentum principle was proposed to dominantly express outlet deviation angle of turbomachine stator by fluid velocity distribution. The stator of some type hydraulic torque converter was taken as a turbomachine stator, and the whole flow passage models of the stators with CFD simulation design parameters were built. The effects of the parameters on outlet deviation angle were analysed respectively by CFD simulation and dominantly expression of outlet deviation angle. Response surface function of outlet deviation angle was constructed by the effects of 
the parameters on outlet deviation angle and CFD simulation data of orthogonal experiment. The average deviation of the response surface function was proved 0.404 degree by CFD simulation data of confirmatory models.

\section{Equivalent Moment of Momentum Principle}

The classical turbomachine design method is based on one-dimension flow theory. According to one-dimension flow theory, a value is chosen as the outlet flow angle instead of the flow angles of all particles. But, the outlet flow angle and the flow angles of all particles must be equivalent. (The particle maybe distributes along stream wise direction or circumferential direction)

Among all of the physical quantities in turbomachine, the moment of every impeller is the macroscopic output physical quantity. The moment is determined by the moment of momentum [1]. So the moment of momentum is chosen as the equivalent principle of the outlet flow angle and the flow angles of all particles. It's called the equivalent moment of momentum principle in the paper.

The moment of momentum can be expressed by all the fluid particles as:

$$
\boldsymbol{L}=\sum_{i=1}^{n} \boldsymbol{r}_{i} \times m_{i} \boldsymbol{v}_{i}
$$

where $L$ identifies moment of momentum; $\boldsymbol{r}$ identifies radius; $m$ identifies mass; $\boldsymbol{v}$ identifies velocity vector; subscript $i$ identifies particle ${ }^{i}$.

The direction of shaft is defined as direction $z$, then, the direction of moment of momentum is in direction $z$. The coordinate of fluid particle $i$ is defined as $\left(x_{i}, y_{i}, z_{i}\right)$, and the velocity component in each direction is $v_{i x}, v_{i y} \cdot v_{i z}$, respectively. Then, it can be derived that:

$$
\left|\boldsymbol{r}_{i} \times m_{i} \boldsymbol{v}_{i}\right|=m_{i}\left(x_{i} v_{i y}-y_{i} v_{i x}\right)
$$

The moment of momentum can also be expressed by radius in the middle flow line, flow velocity, and total mass as:

$$
\boldsymbol{L}=\boldsymbol{R} \times m \boldsymbol{v}
$$

where $\boldsymbol{R}$ identifies radius vector in the middle flow line.

According to the definition of multiplication cross:

$$
|\boldsymbol{R} \times m \boldsymbol{v}|=m|\boldsymbol{R}||\boldsymbol{v}| \sin \alpha
$$

where the $\alpha$ is the angle between $\boldsymbol{R}$ and $\boldsymbol{v}$, as showed in figure 1 .

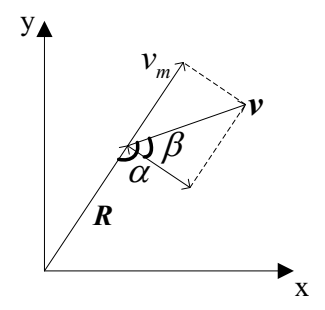

Fig. 1. Vector triangle of stator 
According to one-dimension flow theory, vector triangle of stator can be showed as Fig. 1. Flow angle based on one-dimension flow theory can be expressed as

$$
\sin \beta=\frac{\left|\boldsymbol{v}_{m}\right|}{|\boldsymbol{v}|}
$$

where $\beta$ identifies flow angle based on one-dimension flow theory; $\boldsymbol{v}_{m}$ identifies normal velocity component.

It can be derived in Fig.1 that the relationship between $\alpha$ and $\beta$ can be expressed as

$$
\alpha=\beta+\frac{\pi}{2}
$$

According to one-dimension flow theory, normal velocity component can be expressed by flow rate and normal area as

$$
\left|\boldsymbol{v}_{m}\right|=\frac{Q}{F_{m}}
$$

where $Q$ identifies flow rate; $F_{m}$ identifies normal area.

For fluid, the mass in micro time $\mathrm{t}$ can be expressed by flow rate, density and time as

$$
m_{i}=\rho_{i} Q_{i} t
$$

where $\rho$ identifies density.

The mass of all particles is equal to the total mass. So, it can be derived that

$$
m=\sum_{i=1}^{n} m_{i}
$$

The relationship among the flow angle, blade angle and deviation angle is

$$
\Delta \beta=\beta-\beta_{y}
$$

where $\beta_{y}$ identifies blade angle; $\Delta \beta$ identifies deviation angle.

According to the equivalent moment of momentum principle, formula (1) is equal to formula (3). According to the formulas above, the deviation angle can be expressed as

$$
\Delta \beta=\arctan \left(\frac{|\boldsymbol{R}| Q \sum_{i=1}^{n} \rho_{i} Q_{i}}{F_{m} \sum_{i=1}^{n} \rho_{i} Q_{i}\left(x_{i} v_{i y}-y_{i} v_{i x}\right)}\right)-\beta_{y}
$$

\section{Simulation Models for CFD}

The main research contains of computational fluid dynamics (CFD) is to calculate the flow field with computer. The essence method of CFD is iterative calculation. A lot of studies have proved that the precision of CFD is high. A platform experiment of some type hydraulic torque converter has been done and compared to CFD simulation. As shown in figure 
2 , the average relative errors of torque ratio, efficiency and pump capacity are $0.86 \%, 0.47 \%$ and $1.02 \%$. The reliability of CFD result is proved.

Besides the high precision, another characteristic of CFD is that almost all of the physical quantities can be observed. If CFD is used to calculate the flow field in turbomachine, the physical quantities in formula 11, such as normal area, flow rate, density, velocity and particle coordinate can be observed. So CFD is chosen as the research method.

When the turbomachine is under design, the blade angles of the impellers are the design variables. Circulating flow rate is a very important physical quantity in turbomachine and it has effect on the turbomachine design. Therefore, the simulation models with different inlet blade angles, different outlet blade angles and different flow rates need to be built for CFD.

The inlet impact angle of simulation model is zero, as the turbomachine is under design. Revolving spiral flow passage model was built to control the inlet impact angle.

The stator of some type hydraulic torque converter is taken as a turbomachine stator. The whole flow passage simulation models of the stator with different flow rates, inlet and outlet blade angles and zero inlet impact angles were built for CFD simulation. One of the models is shown in figure 3.

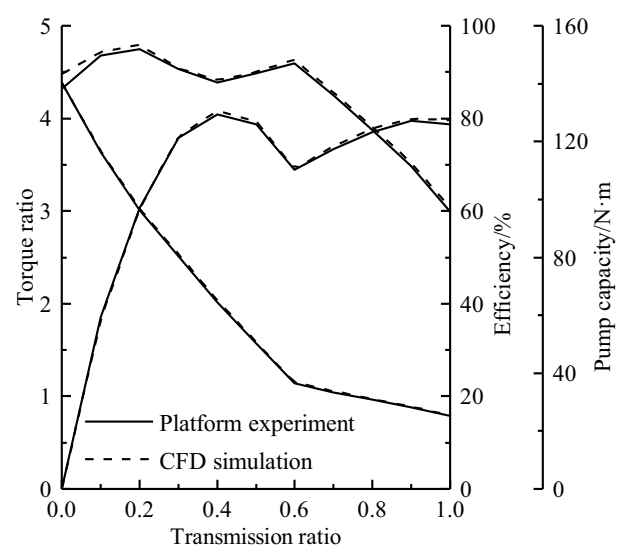

Fig. 2. Comparison of experiment and CFD simulation.

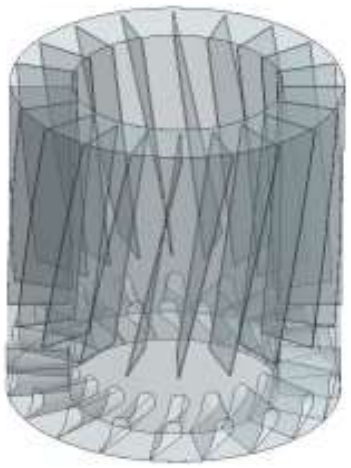

Fig. 3. One of the models for CFD simulation.

\section{Effect of Design Parameter on Outlet Blade Angle}

\subsection{Effect of Inlet Blade Angle on Outlet Blade Angle}

The method to analyse the effect of inlet blade angle on outlet deviation angle is to build some models of which the outlet blade angles and flow rates are the same, but the inlet blade angles are different. CFD simulation results of the models can be got and calculated to analyse the effect.

The inlet blade angle and outlet blade angle of the type hydraulic torque converter stator and the flow rate range are $90 \mathrm{deg}, 35 \mathrm{deg}$ and $0.043-0.149 \mathrm{~m} 3 / \mathrm{s}$.

The design range of inlet blade angle is 20 degree [1]. So, four models need to be built to analyse the effect of inlet blade angle. According to table 1, the outlet blade angle and the flow rates of the models are 35 degree and $0.097 \mathrm{~m} 3 / \mathrm{s}$. The inlet blade angles of the four models are 80 degree, 85 degree, 95 degree and 100 degree respectively.

According the parameters, four models can be built and simulation parameters for CFD can be set. Outlet deviation angles can be calculated by formula 11 and the physical quantities observed from simulation result. The corresponding relationship between inlet blade angle and outlet deviation angle is shown in figure 4 . 


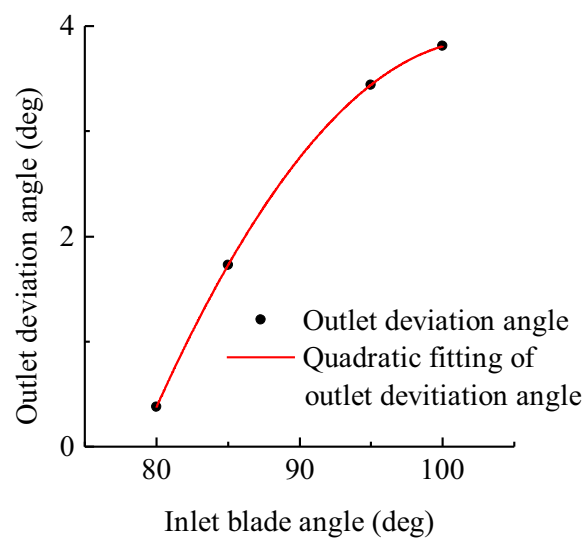

Fig. 4. Outlet deviation angle along inlet blade angle.

It can be seen that, the relationship between inlet blade angle and outlet deviation angle is almost quadric. Quadratic fitting is taken to fit the points. The Residual Sum of Squares (RSS) is $1.79 \times 10-6$. It can be concluded that the relationship between inlet blade angle and outlet deviation angle is almost quadric.

\subsection{Effect of Outlet Blade Angle on Outlet Blade Angle}

The method to analyse the effect of outlet blade angle on outlet deviation angle is similar to that of inlet blade angle. The design range of outlet blade angle is also 20 degree [1]. So, another five models need to be built to analyse the effect of outlet blade angle. According to table 1, the inlet blade angle and the flow rates of the models are 90 degree and $0.097 \mathrm{~m} 3 / \mathrm{s}$. The outlet blade angles of the five models are 25 degree, 30 degree, 35 degree, 40 degree and 45 degree respectively.

According the parameters, five models can be built and simulation parameters for CFD can be set. The outlet deviation angles of stator models with different outlet blade angles can be got in the same way above. The corresponding relationship between outlet blade angle and outlet deviation angle is shown in figure 5 .

It can be seen that, the relationship between outlet blade angle and outlet deviation angle is almost linear. Linear fitting is taken to fit the points. The RSS is $7.83 \times 10-2$. It can be concluded that the relationship between outlet blade angle and outlet deviation angle is almost linear.

\subsection{Effect of Flow Rate on Outlet Blade Angle}

The method to analyse the effect of flow rate on outlet deviation angle is similar to that of inlet blade angle. When the turbomachine works normally, the flow rate range is displayed in table 1. So, another five models need to be built to analyse the effect of flow rate. According to table 1, the inlet blade angle and the outlet blade angle of the models are 90 degree and 35 degree. The flow rates of the five models are $0.04 \mathrm{~m} 3 / \mathrm{s}, 0.069 \mathrm{~m} 3 / \mathrm{s}, 0.097 \mathrm{~m} 3 / \mathrm{s}, 0.126 \mathrm{~m} 3 / \mathrm{s}$ and $0.154 \mathrm{~m} 3 / \mathrm{s}$, respectively.

According the parameters, five models can be built and simulation parameters for CFD can be set. The outlet deviation angles of stator models with different flow rates can be got in the same way above. The relationship between flow rate and outlet deviation angle is shown in Fig. 6. 


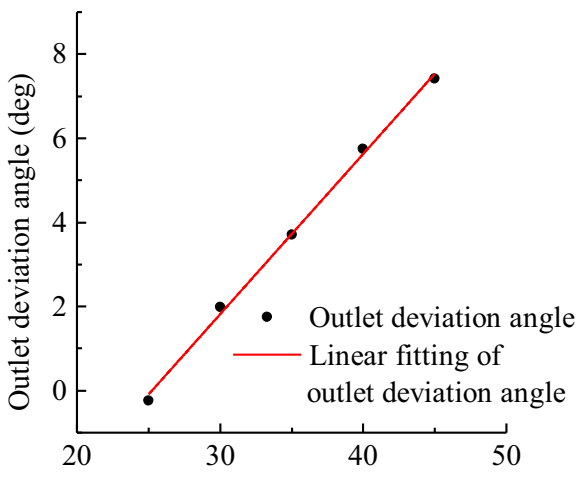

Outlet blade angle (deg)

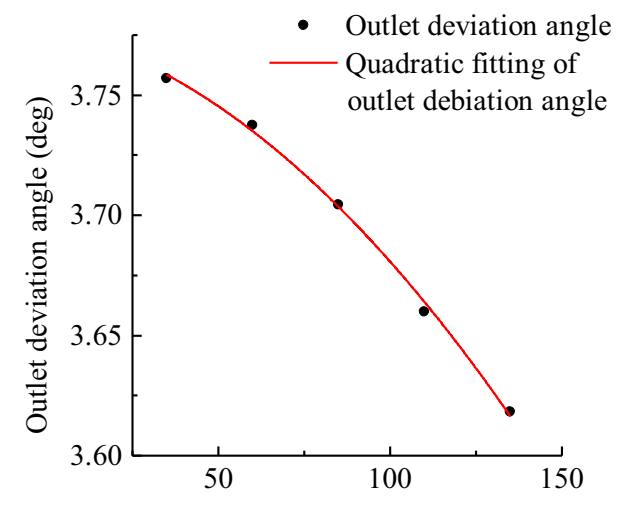

Flow rate $\left(\mathrm{m}^{3} / \mathrm{s}\right)$

Fig. 5. Outlet deviation angle along outlet blade angle.

Fig. 6. Outlet deviation angle along flow rate.

It can be seen that, the relationship between flow rate and outlet deviation angle is almost quadric. Quadratic fitting is taken to fit the points. The RSS is $3.01 \times 10-5$. It can be concluded that the relationship between flow rate and outlet deviation angle is almost quadric.

\section{Response Surface Function}

\subsection{Orthogonal Experiment}

According to the analyses above, the relationships between inlet blade angle, outlet blade angle, flow rate and outlet deviation angle are got. That means the response surface function form of outlet deviation angle is determined, but the coefficients of the function aren't obtained. It can be expressed as:

$$
\begin{aligned}
\Delta \beta= & c_{1}+c_{2} \beta_{y 1}+c_{3} \beta_{y 2}+c_{4} Q+c_{5} \beta_{y 1}{ }^{2}+c_{6} \beta_{y 1} \beta_{y 2}+c_{7} \beta_{y 1} Q+c_{8} \beta_{y 2} Q+c_{9} Q^{2} \\
& +c_{10} \beta_{y 1}{ }^{2} \beta_{y 2}+c_{11} \beta_{y 1}{ }^{2} Q+c_{12} \beta_{y 1} \beta_{y 2} Q+c_{13} \beta_{y 1} Q^{2}+c_{14} \beta_{y 2} Q^{2} \\
& +c_{15} \beta_{y 1}{ }^{2} \beta_{y 2} Q+c_{16} \beta_{y 1}{ }^{2} \beta_{y 2}{ }^{2}+c_{17} \beta_{y 1} \beta_{y 2} Q^{2}+c_{18} \beta_{y 1}{ }^{2} \beta_{y 2} Q^{2}
\end{aligned}
$$

where $C$ identifies undetermined coefficients; subscript 1 and 2 identify inlet and outlet separately.

An equation can be determined by CFD simulation data of one model with a group of CFD simulation design parameter. If there are enough models for CFD simulation, there will be enough equations. When the quantity of equations is bigger than or equal to the quantity of coefficients, the least squares solution of equations can be got. The values of the least squares solution are the values of coefficients.

According to formula 12 , the quantity of coefficients is 18 . So, at least 18 equations are needed. That means at least 18 models with different groups of CFD simulation design parameter need to be built for CFD simulation.

According to formula 12 , the quantity of variables is 3 . For each variable, five values can be chosen. The values of the variables should distribute averagely. Values chosen by orthogonal experiment distribute averagely [8]. So, orthogonal experiment can be chosen to choose the values. Three factors and five levels orthogonal experiment is carried out. According to the orthogonal experiment table L25 (56) [8], 25 groups of parameters can be decided.

\subsection{Response Surface Function}

According to the 25 groups of parameters, 25 models can be built for CFD. Outlet deviation angels of the models can be calculated by formula 11 and fluid velocity distribution obtained from CFD simulation. According to CFD simulation design parameters and outlet deviation angles, coefficients can be got. 


\section{Verification}

The accuracy of the response surface function needs to be verified to prove the function can reflect the real relationship among outlet deviation angle and design parameters.

In order to verify the accuracy of the response surface function, another seven models with different groups of design parameter are built. The parameters are random and in the design range.

CFD simulation of the seven models can be done, and outlet deviation angles can be calculated by formula 11 and fluid velocity distribution obtained from CFD simulation. Outlet deviation angles can also be calculated by the response surface function. The results are shown in figure 7.

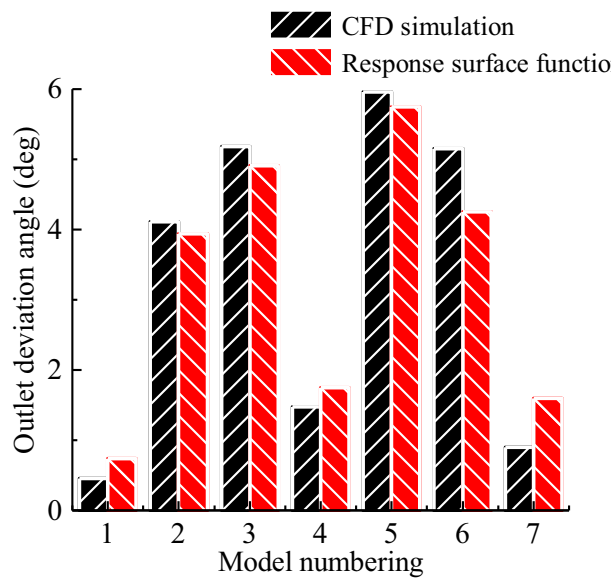

Fig.7. Contrast of outlet deviation angles calculated by CFD simulation and response surface function

It can be seen from figure 7 that the trends of the two kinds of results are the same. The average deviation of the two kinds of results is 0.404 degree.

The deviation of outlet deviation angle causes deviation of moment of momentum. The formula calculating moment of momentum with outlet deviation angle is derived by formula 3-6 and 10 .

$$
|\boldsymbol{L}|=m|\boldsymbol{R}| \frac{\left|\boldsymbol{v}_{m}\right|}{\tan \left(\Delta \beta+\beta_{y}\right)}
$$

As introduced above, design range of outlet blade angle is between 25 degrees to 45 degrees [1]. It can be seen from Fig. 7 that the average outlet deviation angle is about 3 degrees. So, the average error of moment of momentum caused by the 0.404 degree deviation of outlet deviation angle is $1.43 \% \sim 1.71 \%$.

That means the accuracy of formula quantitatively calculating outlet deviation angle in design range can satisfy the engineering demand.

\section{Conclusion}

In this paper, the equivalent moment of momentum principle was proposed according to the macroscopic output physical quantity of turbomachine. Outlet deviation angle dominantly expressed by fluid velocity distribution was derived based on the principle under the condition of certain CFD simulation parameter.

The stator of some type hydraulic torque converter was taken as a turbomachine stator. The effects of CFD simulation parameters on outlet deviation angle were analysed respectively by CFD simulation and dominantly expression of outlet deviation angle. Response surface function of outlet deviation angle was constructed by the effects of the parameters on outlet deviation angle and CFD simulation data of orthogonal experiment. 
The average deviation of the response surface function was proved 0.404 degree by CFD simulation data of confirmatory models. The average error of moment of momentum in design range was $1.43 \% \sim 1.71 \%$. The accuracy of the response surface function could satisfy the engineering demand in design range.

\section{Acknowledgements}

Financial support for this investigation was received from the major science and technology achievements transformation projects of 2012 .

\section{References}

1. Willi B. Stömungsmaschinen (Bere-chnung und Konstruktion). Vogel Press, West Germany (1980).

2. Kluß D, Wiedermann A, Stoff H. Effect of Wakes and Secondary Flow on Re-attachment of Turbine Exit Annular Diffuser Flow. Journal of Turbomachinery, 131, 4 (2009):041012- 1-12.

3. Vassiliev V,Irmisch S,Abdel W S, et al. Impact of the Inflow Conditions on the Heavy-Duty Gas Turbine Exhaust Diffuser Performance. Journal of Turbomachinery, (2010): 1401-1412.

4. Tan M G, Guo B, Liu H L, et al. Investigation of radial force and hydraulic performance in a centrifugal pump with different guide vane outlet angle. 17, 6 (2015): 3247-3260.

5. Hayder M M A. Wake Formation from a Pair of Circular Cylinders Traversing Between Small- and Large-Incidence Flow Regimes. Journal of Fluids Engineering, 134, 1 (2012): 011204-1-12.

6. Padzillah M H, Rajoo S, Yang M, et al. Influence of Pulsating Flow Frequencies towards the Flow Angle Distributions of an Automotive Turbocharger Mixed-flowturbine. Energy Conversion and Management, 98, 1 (2015):449-462.

7. Nishi Y, Fukutomi J. Effect of Blade Outlet Angle on Unsteady Hydrodynamic Force of Closed-Type Centrifugal Pump with Single Blade. International Journal of Rotating Machinery, (2014): 1-16.

8. Taguchi G, Yokoyama T. Experiment Design Method Textbook. Japanese Standards Association, Japan (1981). 